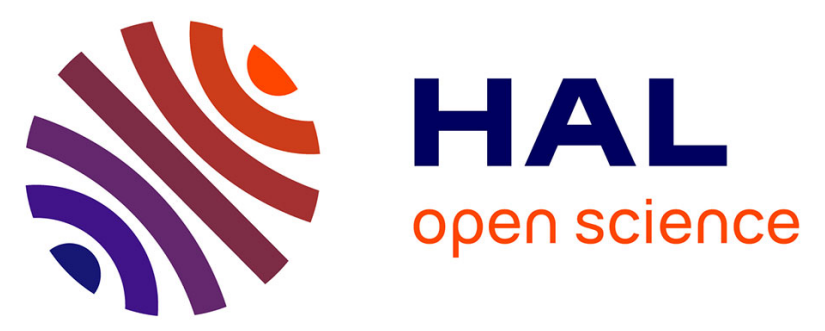

\title{
A novel classification method for prediction of rectal bleeding in prostate cancer radiotherapy based on a semi-nonnegative ICA of 3D planned dose distributions
} Julie Coloigner, Aureline Fargeas, Amar Kachenoura, Lu Wang, Gael Dréan, Caroline Lafond, Lotfi Senhadji, Renaud de Crevoisier, Oscar Acosta, Laurent Albera

\section{To cite this version:}

Julie Coloigner, Aureline Fargeas, Amar Kachenoura, Lu Wang, Gael Dréan, et al.. A novel classification method for prediction of rectal bleeding in prostate cancer radiotherapy based on a seminonnegative ICA of 3D planned dose distributions. IEEE Journal of Biomedical and Health Informatics, 2015, 9 (3), pp.1168-1177. 10.1109/JBHI.2014.2328315 . hal-01011843

\author{
HAL Id: hal-01011843 \\ https://hal.science/hal-01011843
}

Submitted on 24 Jun 2014

HAL is a multi-disciplinary open access archive for the deposit and dissemination of scientific research documents, whether they are published or not. The documents may come from teaching and research institutions in France or abroad, or from public or private research centers.
L'archive ouverte pluridisciplinaire HAL, est destinée au dépôt et à la diffusion de documents scientifiques de niveau recherche, publiés ou non, émanant des établissements d'enseignement et de recherche français ou étrangers, des laboratoires publics ou privés. 


\title{
A novel classification method for prediction of rectal bleeding in prostate cancer radiotherapy based on a semi-nonnegative ICA of 3D planned dose distributions
}

\author{
Julie Coloigner, Auréline Fargeas, Amar Kachenoura, Lu Wang, Gaël Dréan, Caroline Lafond, Lotfi Senhadji, \\ Senior member, IEEE, Renaud de Crevoisier, Oscar Acosta and Laurent Albera, Senior member, IEEE
}

\begin{abstract}
The understanding of dose/side-effects relationships in prostate cancer radiotherapy is crucial to define appropriate individual's constraints for the therapy planning. Most of the existing methods to predict side effects do not fully exploit the rich spatial information conveyed by the three-dimensional planned dose distributions. We propose a new classification method for three-dimensional individuals' doses, based on a new semi-nonnegative ICA algorithm to identify patients at risk of presenting rectal bleeding from a population treated for prostate cancer. The method first determines two bases of vectors from the population data: the two bases span vector subspaces, which characterize patients with and without rectal bleeding, respectively. The classification is then achieved by calculating the distance of a given patient to the two subspaces. The results, obtained on a cohort of 87 patients (at two years follow-up) treated with radiotherapy, showed high performance in terms of sensitivity and specificity.
\end{abstract}

Index Terms-semi-nonnegative ICA algorithm, feature extraction, prostate cancer, rectal bleeding, side effects, radiotherapy, classification.

\section{INTRODUCTION}

Modern RadioTherapy (RT) techniques, such as Intensity Modulated RT (IMRT) and Image Guided RT (IGRT), may allow the increase of dose delivered to the target volume (tumor) [1] but at the expense of the risk of toxicity since neighboring organs may also be over-irradiated [2]-[4]. In the case of prostate cancer, the considered organs at risk are the bladder and the rectum for which the toxicity events may vary in time and complexity. For instance, rectal bleeding, stool loosing and/or urinary incontinence can arise several years after the end of the treatment. Hence, being able to identify a patient who likely will suffer from toxicity events at the early planning stage is of paramount importance in terms of treatment optimization. Understanding the dosetoxicity relationships is, thus, a central question to manage the therapy by selecting appropriate constraints at the inverse planning step in IMRT. Most of the current methods addressing

Julie Coloigner, Auréline Fargeas, Amar Kachenoura, Lu Wang, Gaël Dréan, Lotfi Senhadji, Oscar Acosta and Laurent Albera are with INSERM, U1099, Rennes, F-35000, France; and with Université de Rennes 1, LTSI, Rennes, F-35000, France. julie.coloigner@gmail.com

Caroline Lafond and Renaud de Crevoisier are with INSERM, U1099, Rennes, F-35000, France; and with Université de Rennes 1, LTSI, Rennes, F-35000, France and Département de Radiotherapie, Centre Eugène Marquis, Rennes, F-35000, France. the dose-toxicity relationship issues are based on the ThreeDimensional planned Dose Distribution (3DpDD) via the Dose-Volume Histograms (DVHs) [5] by using radiobiological Normal Tissue Complication Probability (NTCP) models [6][9]. In Prostate Cancer RadioTherapy (PCRT) context, different studies have shown a significant correlation between dose, volume and rectal toxicity [10]-[17]. Nevertheless, these studies present some limitations since they have been solely based on the DVHs: i) the use of DVHs require large databases for the estimation of population specific parameters, ii) different 3DpDD may have similar DVH, and iii) methods based on DVHs lack spatial accuracy, since they considered the organs as having homogeneous radio-sensitivity.

Recently, methods have been proposed which addressed the use of Three-Dimensional (3D) voxel information. Buettner et al. [18], [19] proposed a parameterized representation of the 3DpDD to describe its geometrical properties, such as the eccentricity and its lateral and longitudinal extents. After a first feature extraction step, a formal classification is performed using Support Vector Machine (SVM). However, in this method, there is still a preceding individual feature computation step, which does not jointly use the voxel information across the whole population. Exploiting the rich voxel information may be hampered by the large inter-individual anatomical variability. If all the voxel dose information are to be analyzed across the population, a previous anatomical alignment and dose mapping into a common spatial coordinate system is performed. This is particularly challenging due to the poor soft-tissue contrast, large inter-individual variability, and differences in bladder and rectum filling [20], but some registration methods have been proposed tackling this problem [21]. Following this idea, population analysis and voxelwise comparison of 3DpDD has been performed in [22], [23]. Although these approaches enable the identification of 3D anatomical patterns which may be responsible for toxicity, they do not perform any formal classification or a prediction of toxicity for a given individual. Thus, new methods aimed at jointly taking advantage of the $3 \mathrm{DpDD}$, that reveal the subtle correlation between local dose and toxicity at a voxel level to classify patients at risk, are still to be devised.

Assuming perfectly aligned 3D images, the Principal Component Analysis (PCA) can be used to extract features for classification from voxel information by considering each voxel as a decorrelated observation. For instance, PCA has been 
performed for feature extraction and dimensionality reduction in the identification of Alzheimer's disease [24] using positron emission tomography images. PCA computes an orthogonal basis by maximizing the variance of the coordinates of each 3D individual image in the subspace spanned by the basis vectors. In the context of rectal bleeding, PCA is used to analyze non-rigidly registered dose distributions and classify bleeding patients [25], [26]. This work aimed at identifying one basis of orthogonal vectors from 3DpDD of rectal bleeding and non rectal bleeding patients, allowing good classification results. However, PCA is limited by the orthogonal constraints defined in the problem formulation. This constraint can be relaxed by using more statistical information from 3DpDD, such as the mutual independence of the signal of interest (sources) [27]. This leads to the Independent Component Analysis (ICA) concept [28]. ICA has been successfully used for extracting linear features combinations [29], [30]. Moreover, in some blind source separation applications, such as face recognition [31] or tumors classification [32], [33], the components of the mixing matrix can represent pixels and they are indeed positive. In order to improve the extraction quality, this nonnegativity property can be exploited, giving rise to what we call hereafter the Semi-Nonnegative ICA (SN-ICA).

In our context, the components of the mixing matrix correspond also to the intensity of the dose such as a positive value (which means delivered energy per mass unit at each voxel). In this paper, we propose a new classification procedure, based on a new SN-ICA algorithm, to characterize rectal bleeding on groups of patients treated by radiotherapy. More precisely, this procedure aims at determining two bases from 3DpDD: the first basis characterizes the rectal bleeding and the second one corresponds to the non rectal bleeding patients. The classification is achieved by projecting a new 3D individual planned Dose Distributions (pDD) onto both subspaces spanned by the two vector bases. The new patient is thus classified using the smaller distance to both subspaces. Tests on real clinical database of 87 patients (13 of them presented rectal bleeding within a 2-year follow-up) and the comparison with some classical methods showed high performance of the proposed method in terms of sensitivity and specificity. The obtained results revealed spatial relationships between the 3DpDD and treatment outcome, paving a way for the prediction of toxicity in PCRT.

\section{THE CLASSIFICATION PROCEDURE}

Our classification procedure involves three steps, namely the preprocessing step, the training step and the classification step, which are summarized in figure 1 .

1) Preprocessing step: It is divided into two sub-steps: i) data registration and ii) data structuring.

a) Data registration: In order to perform a voxel based statistical analysis, an accurate mapping of the dose distribution received by the rectum is required. Patient's planning $\mathrm{CT}$ and dose distributions are non-rigidly registered into a common coordinate system. This non-rigid registration approach advantageously combines information available at the planning step, namely the 3D anatomical data (individual CTs) and organ manual delineations. Thus, euclidean distance maps from organs boundaries are built and combined with CT scans to perform a non-rigid demons registration method based warping as described in [22]. Regarding the single template, it was selected as the individual closest to all of the remaining individuals. This typical individual was found by affinity propagation clustering among a subgroup of randomly selected patients from the whole database [22]. In our study we focus on rectal bleeding and only the registered 3DpDD within the rectum is considered.

b) Data structuring: Each individual's 3DpDD is arranged in a vector by vertically concatenating the rows of all slices. Thus, we obtained an $\left(N \times P^{(1)}\right)$ matrix $\mathbf{X}^{(1)}$ for patients with rectal bleeding and an $\left(N \times P^{(2)}\right)$ matrix $\mathbf{X}^{(2)}$ for those without rectal bleeding: $P^{(1)}, P^{(2)}$ and $N$ represent the number of individuals with rectal bleeding, the number of individuals without rectal bleeding and the number of voxels, respectively.

2) Training step: In this step we first identify from the data two vector bases, $\boldsymbol{A}^{(1)}=\left(\boldsymbol{a}_{1}^{(1)}, \ldots, \boldsymbol{a}_{F^{(1)}}^{(1)}\right)$ and $\boldsymbol{A}^{(2)}=\left(\boldsymbol{a}_{1}^{(2)}, \ldots, \boldsymbol{a}_{F^{(2)}}^{(2)}\right)$, spanning two vector subspaces, $\mathcal{A}^{(1)}$ and $\mathcal{A}^{(2)}$, that characterize patients with and without rectal bleeding, respectively. The dimensions $F^{(i)}(i=\{1,2\})$ are usually lower than $P^{(i)}$. Secondly, we construct from $\mathcal{A}^{(1)}$ and $\mathcal{A}^{(2)}$ two sets of subspaces, called $\varepsilon^{(1)}=\left\{\varepsilon_{1}^{(1)}, \ldots, \varepsilon_{K}^{(1)}\right\}$ and $\varepsilon^{(2)}=\left\{\varepsilon_{1}^{(2)}, \ldots, \varepsilon_{L}^{(2)}\right\}$. More precisely, we respectively built from $\boldsymbol{A}^{(1)}$ and $\boldsymbol{A}^{(2)}$ two sets $\mathcal{I}^{(1)}=\left\{\boldsymbol{E}_{1}^{(1)}, \ldots, \boldsymbol{E}_{K}^{(1)}\right\}$ and $\mathcal{I}^{(2)}=\left\{\boldsymbol{E}_{1}^{(2)}, \ldots, \boldsymbol{E}_{L}^{(2)}\right\}$ (where $\boldsymbol{E}_{k}^{(1)}, k \in\{1, \ldots, K\}$ is a sub-basis of rank $R^{(1)}$, with $R^{(1)} \leq F^{(1)}$, and $\boldsymbol{E}_{\ell}^{(2)}, \ell \in$ $\{1, \ldots, L\}$, is of rank $R^{(2)}$, with $\left.R^{(2)} \leq F^{(2)}\right)$. It is noteworthy that each subspace $\varepsilon_{j}^{(i)}$ is spanned by a vector subbasis $\boldsymbol{E}_{j}^{(i)}$. Two key questions, that will be discussed in details in sections III-B and II-3, arise: i) the calculation of $\boldsymbol{A}^{(1)}$ and $\boldsymbol{A}^{(2)}$, and ii) the choice of the optimal subbases $\boldsymbol{E}_{k_{\mathrm{opt}}}^{(1)}$ and $\boldsymbol{E}_{\ell_{\mathrm{opt}}}^{(2)}$, containing the more informative features of $\boldsymbol{A}^{(1)}$ and $\boldsymbol{A}^{(2)}$, that better divide the patients in rectal and non rectal bleeding groups.

3) Classification step: In order to classify a new patient belonging to the testing set, its $3 \mathrm{D}$ individual $\mathrm{pDD}, \boldsymbol{x}_{\text {new }}$, is orthogonally projected onto all subspaces of $\varepsilon^{(1)}$ and $\varepsilon^{(2)}$, characterizing the two groups (patients with and without rectal bleeding) as follows:

$$
\begin{gathered}
\boldsymbol{g}_{k}^{(1)}=\Delta_{\varepsilon_{k}^{(1)}}^{\perp} \boldsymbol{x}_{n e w}(1 \leq k \leq K) \\
\boldsymbol{g}_{\ell}^{(2)}=\Delta_{\boldsymbol{\varepsilon}_{\ell}^{(2)}}^{\perp} \boldsymbol{x}_{n e w}(1 \leq \ell \leq L)
\end{gathered}
$$

where $\boldsymbol{\Delta}_{\varepsilon_{k}^{(1)}}^{\perp}=\boldsymbol{E}_{k}^{(1)}\left(\boldsymbol{E}_{k}^{(1)_{\top}} \boldsymbol{E}_{k}^{(1)}\right)^{-1} \boldsymbol{E}_{k}^{(1)_{\top}}$ and $\boldsymbol{\Delta}_{\varepsilon_{\ell}^{(2)}}^{\perp}=$ $\boldsymbol{E}_{\ell}^{(2)}\left(\boldsymbol{E}_{\ell}^{(2) \top} \boldsymbol{E}_{\ell}^{(2)}\right)^{-1} \boldsymbol{E}_{\ell}^{(2) \top}$. Afterward, the euclidean distances between $\boldsymbol{x}_{\text {new }}$ and its orthogonal projections are computed by:

$$
\begin{gathered}
d_{k}^{(1)}=\left\|\boldsymbol{x}_{n e w}-\boldsymbol{g}_{k}^{(1)}\right\|(1 \leq k \leq K) \\
d_{\ell}^{(2)}=\left\|\boldsymbol{x}_{n e w}-\boldsymbol{g}_{\ell}^{(2)}\right\|(1 \leq \ell \leq L)
\end{gathered}
$$



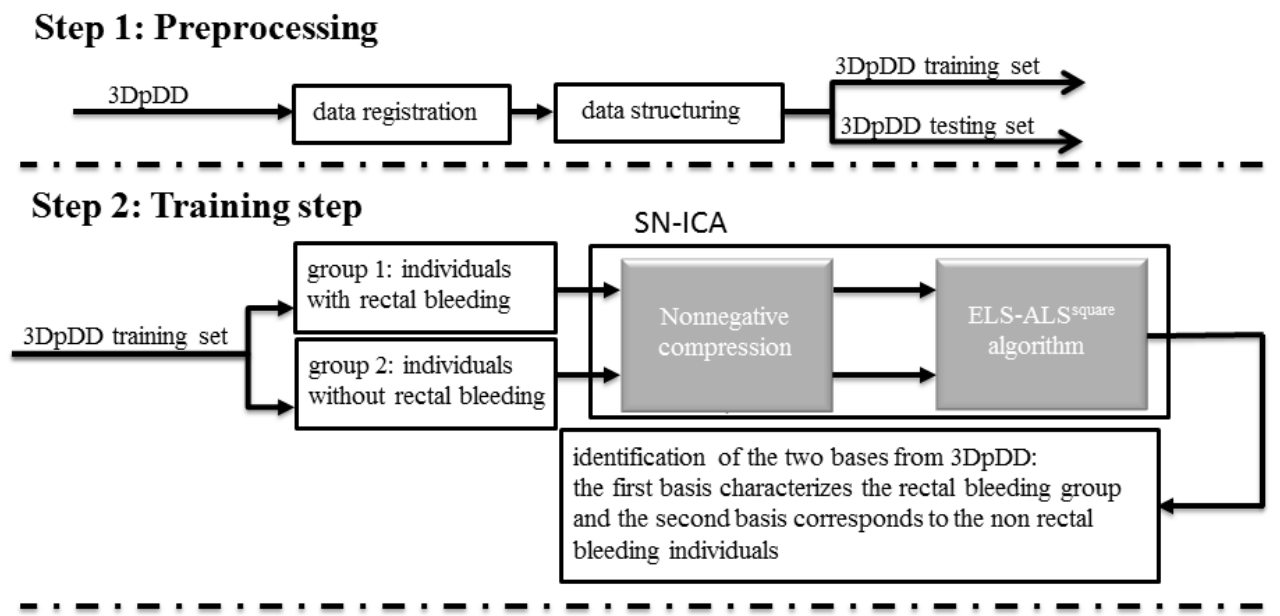

Step 3: Classification step

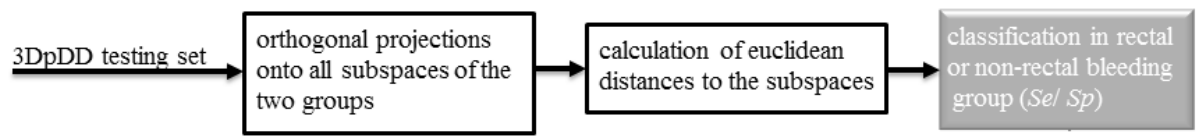

Fig. 1. The three steps of the classification procedure: 1) the preprocessing step: the data are registered and structured, 2) the training step: the two vector bases spanning the rectal and non rectal bleeding subspaces are calculated, and 3) the classification step: the new 3DpDD is orthogonally projected onto both subspaces and the new patient is classified in the closest group.

To select the optimal couple $\left(\boldsymbol{E}_{k_{\mathrm{opt}}}^{(1)}, \boldsymbol{E}_{\ell_{\mathrm{opt}}}^{(2)}\right)$, we maximize the following criterion:

$$
\left(d_{k_{\mathrm{opt}}}^{(1)}, d_{\ell_{\mathrm{opt}}}^{(2)}\right)=\underset{\left(d_{k}^{(1)}, d_{\ell}^{(2)}\right)}{\arg \max }\left(d_{k}^{(1)}-d_{\ell}^{(2)}\right)
$$

with $1 \leq k \leq K$ and $1 \leq \ell \leq L$. Finally, the new patient is classified as belonging to the closest group: if $d_{k_{\text {opt }}}^{(1)}<d_{\ell_{\mathrm{opt}}}^{(2)}$, we will deduce that he belongs to the rectal bleeding group. Otherwise, he is classified as non rectal bleeding patient. It is interesting to note that this step is performed by the euclidean norm and does not require any evolutive classification method.

\section{PROBLEM FORMULATION AND METHOD}

This section presents the new SN-ICA algorithm that will be used to estimate the vector bases $\boldsymbol{A}^{(1)}=\left(\boldsymbol{a}_{1}^{(1)}, \ldots, \boldsymbol{a}_{F^{(1)}}^{(1)}\right)$ and $\boldsymbol{A}^{(2)}=\left(\boldsymbol{a}_{1}^{(2)}, \ldots, \boldsymbol{a}_{F^{(2)}}^{(2)}\right)$, that characterize patients with and without rectal bleeding, respectively. In the sequel, we present the algorithm in general case, namely to estimate the basis $\boldsymbol{A}^{(i)}$, whatever $i \in\{1,2\}$.

\section{A. Problem formulation}

As explained in section II-1, $\boldsymbol{X}^{(i)}$ contains the 3DpDD of all patients belonging to the $i^{t h}$ group (each individual's 3DpDD is arranged in a column vector). More particularly, assume that each individual 3DpDD, $\boldsymbol{x}^{(i)}[m]=\left[x_{1}^{(i)}[m], \ldots, x_{N}^{(i)}[m]\right]^{T}$, is one realization of a $N$-dimensional random process $\left\{\boldsymbol{x}^{(i)}[m]\right\}$. $\boldsymbol{x}^{(i)}[\mathrm{m}]$ is modeled as an linear combination of basis vectors $\left(\boldsymbol{a}_{1}^{(i)}, \ldots, \boldsymbol{a}_{F^{(i)}}^{(i)}\right)$, whose weights are denoted by, $\boldsymbol{s}^{(i)}[m]=$ $\left[s_{1}^{(i)}[m], \ldots, s_{F^{(i)}}^{(i)}[m]\right]^{T}$. In our context, each component of $\boldsymbol{A}^{(i)}$ represent the intensity value of the dose and it is indeed positive. In addition, we assume that the coordinates of $\boldsymbol{x}^{(i)}[m]$ in basis $\boldsymbol{A}^{(i)}$, namely the elements of $\boldsymbol{s}^{(i)}[m]$, are independent. So, the problem we tackle in this paper can be formulated using the Semi-Nonnegative ICA (SN-ICA) one [34], [35].

Problem 1: Given one realization of a real random vector process $\left\{\boldsymbol{x}^{(i)}[m]\right\}$, find an $\left(N \times F^{(i)}\right)$ matrix $\boldsymbol{A}^{(i)}$ and one realization of a $F^{(i)}$-dimensional source random process $\left\{\boldsymbol{s}^{(i)}[m]\right\}$, such that for each index $m$ :

$\boldsymbol{x}^{(i)}[m]=\boldsymbol{A}^{(i)} \boldsymbol{s}^{(i)}[m]$, where $\boldsymbol{A}^{(i)}$ has nonnegative components and $\boldsymbol{s}^{(i)}[\mathrm{m}]$ has statistically independent components.

\section{B. SN-ICA algorithm}

As depicted in figure 1, the SN-ICA method consists, first, in a nonnegative compression and rank estimation step which aims to reduce the dimension of the observation space. The second step, based on the ELS-ALS square algorithm [34], estimates the bases $\boldsymbol{A}^{(i)},(i \in\{1,2\})$ from the compressed observations.

1) The nonnegative compression and rank estimation: In order to reduce the dimension of the observation space, the nonnegative compression method truncates the $N$-dimensional vector $\boldsymbol{x}^{(i)}[m]$ into a vector $\tilde{\boldsymbol{x}}^{(i)}[m]$ of rank $F^{(i)} \ll N$. The compressed observations are expressed as follows:

$$
\tilde{\boldsymbol{x}}^{(i)}[m]=\boldsymbol{W}^{(i) \top} \boldsymbol{x}^{(i)}[m]
$$


where $\boldsymbol{W}^{(i)}$ of size $\left(N \times F^{(i)}\right)$ is the compression matrix. For the ICA model without nonnegative constraint, the columns of $\boldsymbol{W}^{(i)}$ are the eigenvectors corresponding to the $F^{(i)}$ largest eigenvalues of the covariance matrix $E\left(\boldsymbol{x}^{(i)}[m] \boldsymbol{x}^{(i)}[m]^{\top}\right)-$ $E\left(\boldsymbol{x}^{(i)}[m]\right) E\left(\boldsymbol{x}^{(i)}[m]^{\top}\right)$ and the estimate of the rank, $F^{(i)}$, is determined by the number of eigenvalues not exceedingly close to zero [36]. However, in our model, such a method cannot guarantee the nonnegativity of the compressed basis matrix $\tilde{\boldsymbol{A}}^{(i)}=\boldsymbol{W}^{(i) \top} \boldsymbol{A}^{(i)}$, since generally $\boldsymbol{W}^{(i)}$ is not nonnegative. It is possible to transform $\boldsymbol{W}^{(i)}$ into the nonnegative orthant by column-pair rotations [37], [38].

In practice, the only use of the rotation transformation is not sufficient to guarantee the nonnegativity of $\boldsymbol{W}^{(i)}$. This is done by the introduction of the shearing transformation on the pairs of columns of $\boldsymbol{W}^{(i)}$ (see Appendix A for details). Thus, if $\boldsymbol{W}^{(i)}$ is nonnegative, the compressed matrix $\tilde{\boldsymbol{A}}^{(i)}$ of size $\left(F^{(i)} \times F^{(i)}\right)$ preserves the nonnegativity property of $\boldsymbol{A}^{(i)}$. The rank is also estimated by singular value decomposition [36], as in the common compression. So, the compressed 3D individual pDD vector, $\tilde{\boldsymbol{x}}^{(i)}[\mathrm{m}]$ still follows the SN-ICA model: $\tilde{\boldsymbol{x}}^{(i)}[m]=\tilde{\boldsymbol{A}}^{(i)} \tilde{\boldsymbol{s}}^{(i)}[m]$.

2) The SN-ICA based ELS-ALS $S^{\text {square }}$ algorithm: The SNICA algorithm exploits some interesting properties enjoyed by cumulants in the presence of mixed independent processes [34]. Let $\mathcal{C}_{n_{1}, n_{2}, n_{3}, \tilde{\boldsymbol{x}}^{(i)}}$ and $\mathcal{C}_{n_{1}, n_{2}, n_{3}, n_{4}, \tilde{\boldsymbol{x}}^{(i)}}$ be the entries of the Third Order (TO) and Fourth Order (FO) cumulant arrays, $\mathcal{C}_{3, \tilde{\boldsymbol{x}}^{(i)}}$ and $\mathcal{C}_{4, \tilde{\boldsymbol{x}}^{(i)}}$, respectively, of a zero-mean $F^{(i)}$ dimensional random vector $\left\{\tilde{\boldsymbol{x}}^{(i)}[m]\right\}$. Under the assumptions made in SN-ICA problem and due to the multi-linearity property of cumulants, we have:

$$
\begin{array}{r}
\mathcal{C}_{n_{1}, n_{2}, n_{3}, \tilde{\boldsymbol{x}}^{(i)}}=\sum_{f=1}^{F^{(i)}} \tilde{A}_{n_{1}, f}^{(i)} \tilde{A}_{n_{2}, f}^{(i)} \tilde{A}_{n_{3}, f}^{(i)} \mathcal{C}_{f, f, f, \tilde{\boldsymbol{s}}^{(i)}} \\
\mathcal{C}_{n_{1}, n_{2}, n_{3}, n_{4}, \tilde{\boldsymbol{x}}^{(i)}}=\sum_{f=1}^{F^{(i)}} \tilde{A}_{n_{1}, f}^{(i)} \tilde{A}_{n_{2}, f}^{(i)} \tilde{A}_{n_{3}, f} \tilde{A}_{n_{4}, f}^{(i)} \mathcal{C}_{f, f, f, f, \tilde{\boldsymbol{s}}^{(i)}}
\end{array}
$$

where $\mathcal{C}_{f, f, f, \tilde{\boldsymbol{s}}^{(i)}}$ is the $(f, f, f)$-th element of the TO cumulant array of $\left\{\tilde{\boldsymbol{s}}^{(i)}[m]\right\}, \mathcal{C}_{f, f, f, f, \tilde{\boldsymbol{s}}^{(i)}}$ is the $(f, f, f, f)$-th element of the FO cumulant array of $\left\{\tilde{\boldsymbol{s}}^{(i)}[m]\right\}$ and $F^{(i)}$ is the number of extracted sources. Note that the dimensions of $\mathcal{C}_{3, \tilde{x}^{(i)}}$ and $\mathcal{C}_{4, \tilde{\boldsymbol{x}}^{(i)}}$ are $\left(F^{(i)} \times F^{(i)} \times F^{(i)}\right)$ and $\left(F^{(i)} \times F^{(i)} \times F^{(i)} \times F^{(i)}\right)$, respectively. We propose to merge together the entries of the TO and FO cumulant arrays in the rectangular matrix $T_{\tilde{x}^{(i)}}^{(3,4)}$ of size $\left(F^{(i)}+\left(F^{(i)}\right)^{2} \times\left(F^{(i)}\right)^{2}\right)$. The $\left(i_{1}, i_{2}\right)$-th entry, $T_{i_{1}, i_{2}, \tilde{\boldsymbol{x}}^{(i)}}^{\tilde{\tilde{\boldsymbol{x}}}^{(i)}}$ of $\boldsymbol{T}_{\tilde{\boldsymbol{x}}^{(i)}}^{(3,4)}$ is given by:

$T_{i_{1}, i_{2}, \tilde{\boldsymbol{x}}^{(i)}}^{(3,4)}=\left\{\begin{array}{l}\mathcal{C}_{n_{1}, n_{2}, i_{1}, \tilde{\boldsymbol{x}}^{(i)}} \text { for any } i_{1} \in\left\{1, \ldots, F^{(i)}\right\} \\ \text { with } i_{2}=n_{1}+F^{(i)}\left(n_{2}-1\right) \\ \mathcal{C}_{n_{1}, n_{2}, n_{3}, n_{4}, \tilde{\boldsymbol{x}}^{(i)} \text { with } i_{1}=n_{4}+F^{(i)} n_{3}} \\ \text { and } i_{2}=n_{2}+F^{(i)}\left(n_{1}-1\right) \\ \text { for any } i_{1} \in\left\{F^{(i)}+1, \ldots, F^{(i)}+\left(F^{(i)}\right)^{2}\right\}\end{array}\right.$

By inserting (7) and (8), we obtain the following algebraic structure of the matrix $\boldsymbol{T}_{\tilde{\boldsymbol{x}}^{(i)}}^{(3,4)}$ :

$$
\boldsymbol{T}_{\tilde{\boldsymbol{x}}^{(i)}}^{(3,4)}=\tilde{\boldsymbol{C}}^{(i)}\left(\left(\tilde{\boldsymbol{A}}^{(i)}\right)^{\odot 2}\right)^{\top}+\boldsymbol{V}^{(i)}
$$

with $\tilde{\boldsymbol{C}}^{(i)}=\left[\boldsymbol{C}_{3, \tilde{\boldsymbol{s}}^{(i)}} \tilde{\boldsymbol{A}}^{\top}, \boldsymbol{C}_{4, \tilde{\boldsymbol{s}}^{(i)}}(\tilde{\boldsymbol{A}} \odot \tilde{\boldsymbol{A}})^{\top}\right]^{\top}$ where the matrices $\boldsymbol{C}_{3, \tilde{\boldsymbol{s}}^{(i)}}=\operatorname{diag}\left(\left[\mathcal{C}_{1,1,1, \tilde{\boldsymbol{s}}^{(i)}}, \cdots, \mathcal{C}_{F^{(i)}, F^{(i)}, F^{(i)}, \tilde{\boldsymbol{s}}^{(i)}}\right]\right)$ and

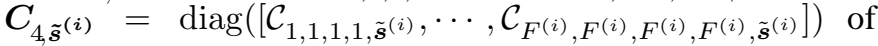
size $\left(F^{(i)} \times F^{(i)}\right)$ are diagonal. The symbol $\odot$ stands for the Khatri-Rao product (column-wise Kronecker product) and we denote: $\left(\tilde{\boldsymbol{A}}^{(i)}\right)^{\odot 2}=\tilde{\boldsymbol{A}}^{(i)} \odot \tilde{\boldsymbol{A}}^{(i)}$. The matrix $\boldsymbol{V}^{(i)}$ represents the model residual. As a way of treating the nonnegativity constraint of the matrix $\tilde{\boldsymbol{A}}^{(i)}$ in the SN-ICA model, one can resort to the square change of variable $\tilde{\boldsymbol{A}}^{(i)}=\left(\tilde{\boldsymbol{B}}^{(i)}\right)^{\diamond 2}$, with $\boldsymbol{B}^{(i)} \in \mathbb{R}^{F^{(i)} \times F^{(i)}}$ (where $\left(\boldsymbol{B}^{(i)}\right)^{\diamond 2}=\boldsymbol{B}^{(i)} \diamond \boldsymbol{B}^{(i)}$ and the symbol $\diamond$ represents the Hadamard product (element-wise product)). The SN-ICA problem is, then, totally characterized by the following objective function:

$$
f\left(\tilde{\boldsymbol{B}}^{(i)}, \tilde{\boldsymbol{C}}^{(i)}\right)=\left\|\boldsymbol{T}_{\tilde{\boldsymbol{x}}^{(i)}}^{(3,4)}-\tilde{\boldsymbol{C}}^{(i)}\left(\left(\left(\tilde{\boldsymbol{B}}^{(i)}\right)^{\diamond 2}\right)^{\odot 2}\right)^{\top}\right\|_{F}^{2}
$$

where the couple of variables $\left(\tilde{\boldsymbol{B}}^{(i)}, \tilde{\boldsymbol{C}}^{(i)}\right)$ belongs to the open set $\mathbb{R}^{\left(F^{(i)}+\left(F^{(i)}\right)^{2}\right) \times F^{(i)}} \times \mathbb{R}^{F^{(i)} \times F^{(i)}}$ and the symbol $\|\cdot\|_{F}$ denotes the Frobenius norm. It is worth noting that the nonnegative constraint is circumvented by means of the square change of variable, leading to unconstrained problem.

a) Alternating Least Squares (ALS) procedure: ALS procedure is proposed, in order to minimize (10), taken into account several advantages, such as the decomposition accuracy, the numerical complexity and the memory requirements [39]. The ALS principle is to reduce the non-linear minimization problem of $f(10)$ to several coupled linear least-squares subproblems. To do so, we choose to alternatively minimize the cost function w.r.t. $\tilde{\boldsymbol{B}}^{(i)}$ and $\tilde{\boldsymbol{C}}^{(i)}$, optimizing w.r.t. one variable while keeping the other one fixed. Then, we solve sequentially, for the iteration $i t$, both following subproblems:

$$
\begin{aligned}
& \tilde{\boldsymbol{B}}_{i t+1}^{(i)}=\underset{\tilde{B}^{(i)}}{\arg \min } f_{B^{(i)}}=\underset{\tilde{B}^{(i)}}{\arg \min }\left\|\boldsymbol{T}_{\tilde{\boldsymbol{x}}^{(i)}}^{(3,4)}-\tilde{\boldsymbol{C}}_{i t}^{(i)}\left(\left(\tilde{\boldsymbol{A}}^{(i)}\right)^{\odot 2}\right)^{\top}\right\|^{2} \\
& \tilde{\boldsymbol{C}}_{i t+1}^{(i)}=\underset{\tilde{C}^{(i)}}{\arg \min } f_{\tilde{C}^{(i)}}=\underset{\tilde{C}^{(i)}}{\arg \min }\left\|\boldsymbol{T}_{\tilde{\boldsymbol{x}}^{(i)}}^{(3,4)}-\tilde{\boldsymbol{C}}^{(i)}\left(\left(\tilde{\boldsymbol{A}}_{i t+1}^{(i)}\right)^{\odot 2}\right)^{\top}\right\|^{2}
\end{aligned}
$$

with $\tilde{\boldsymbol{A}}^{(i)}=\left(\tilde{\boldsymbol{B}}^{(i)}\right)^{\diamond 2}$, and where $f_{\tilde{B}^{(i)}}$ and $f_{\tilde{C}^{(i)}}$ depend only on one free variable, $\tilde{\boldsymbol{B}}^{(i)}$ and $\tilde{\boldsymbol{C}}^{(i)}$, respectively. The solution $\tilde{\boldsymbol{B}}_{i t+1}^{(i)}$ of the first subproblem can be obtained by alternatively solving $f_{B^{(i)}}$ w.r.t. all components of $\tilde{\boldsymbol{B}}^{(i)}$. So, we introduce $f_{\tilde{B}_{m, n}^{(i)}}: \tilde{B}_{m, n}^{(i)} \mapsto f_{B^{(i)}}\left(\tilde{B}^{(i)}\right)$, where $\tilde{B}_{m, n}^{(i)}$ is the $(m, n)$ th component of $\tilde{\boldsymbol{B}}^{(i)}$. The element of $\tilde{\boldsymbol{B}}_{i t+1}^{(i)}$ is achieved by vanishing the derivative denoted $f_{\tilde{B}_{m, n}^{(i)}}^{\prime}$. It is noteworthy that this derivative is a polynomial in variable $\tilde{B}_{m, n}^{(i)}$ and the expression is given by:

$$
f_{\tilde{B}_{m, n}^{(i)}}^{\prime}\left(\tilde{B}_{m, n}^{(i)}\right)=\alpha_{7}\left(\tilde{B}_{m, n}^{(i)}\right)^{7}+\alpha_{3}\left(\tilde{B}_{m, n}^{(i)}\right)^{3}+\alpha_{1} \tilde{B}_{m, n}^{(i)}
$$


with:

$$
\begin{aligned}
\alpha_{1}= & -2\left(\tilde{\boldsymbol{A}}_{i t}^{(i)}\left(\boldsymbol{M}_{i t}^{(i)}+\boldsymbol{N}_{i t}^{(i)}\right)\right)_{n,(n-1) F^{(i)}+m}- \\
& 4\left(\tilde{\boldsymbol{A}}_{i t}^{(i)}\left(\left(\left(\tilde{\boldsymbol{A}}_{i t}^{(i)}\right)^{\top} \tilde{\boldsymbol{A}}_{i t}^{(i)}\right) \diamond\left(\left(\tilde{\boldsymbol{C}}_{i t}^{(i)}\right)^{\top} \tilde{\boldsymbol{C}}_{i t}^{(i)}\right)\right)\right)_{m, n}- \\
& 4 \tilde{A}_{m, n}^{(i)}\left(\left(\left(\tilde{\boldsymbol{C}}_{i t}^{(i)}\right)^{\top} \tilde{\boldsymbol{C}}_{i t}^{(i)}\left(\left(\tilde{\boldsymbol{A}}_{i t}^{(i)}\right)^{\top}\right)^{\diamond 2}\right)+\right. \\
& \left(\left(\tilde{\boldsymbol{A}}_{i t}^{(i)}\right)^{\top} \tilde{\boldsymbol{A}}_{i t}^{(i)}\right)_{n, n}\left(\left(\tilde{\boldsymbol{C}}_{i t}^{(i)}\right)^{\top} \tilde{\boldsymbol{C}}_{i t}^{(i)}\right)_{n, n}- \\
& \left.\left(\tilde{A}_{m, n}^{(i)}\right)^{2}\left(\left(\tilde{\boldsymbol{C}}_{i t}^{(i)}\right)^{\top} \tilde{\boldsymbol{C}}_{i t}^{(i)}\right)_{n, n}\right) \\
& -2 \tilde{\boldsymbol{A}}_{m, n}^{(i)}\left(\boldsymbol{M}_{i t}^{(i)}+\boldsymbol{N}_{i t}^{(i)}\right)_{m,(n-1) F^{(i)}+m} \\
\alpha_{3}= & -2\left(\boldsymbol{M}_{i t}^{(i)}+\boldsymbol{N}_{i t}^{(i)}\right)_{m,(n-1) F^{(i)}+m}+ \\
& 4\left(\left(\tilde{\boldsymbol{A}}_{i t}^{(i)}\right)^{\diamond 2}\left(\tilde{\boldsymbol{C}}_{i t}^{(i)}\right)^{\top} \tilde{\boldsymbol{C}}_{i t}^{(i)}\right)_{m, n}+ \\
& 4\left(\left(\left(\tilde{\boldsymbol{A}}_{i t}^{(i)}\right)^{\top} \tilde{\boldsymbol{A}}_{i t}^{(i)}\right)_{n, n}\left(\left(\tilde{\boldsymbol{C}}_{i t}^{(i)}\right)^{\top} \tilde{\boldsymbol{C}}_{i t}^{(i)}\right)_{n, n}-\right. \\
& 8\left(\tilde{A}_{m, n}^{(i)}\right)^{\diamond 2}\left(\left(\tilde{\boldsymbol{C}}_{i t}^{(i)}\right)^{\top} \tilde{\boldsymbol{C}}_{i t}^{(i)}\right)_{n, n} \\
\alpha_{7}= & 4\left(\left(\tilde{\boldsymbol{C}}_{i t}^{(i)}\right)^{\top} \tilde{\boldsymbol{A}}_{i t}^{(i)}\right)_{n, n}
\end{aligned}
$$

where $\boldsymbol{M}_{i t}^{(i)}=\operatorname{Mat}^{\left(F^{(i)} \times 1,\left(F^{(i)}\right)^{2}\right)}\left(\operatorname{vec}\left(\boldsymbol{T}_{\tilde{\boldsymbol{x}}^{(i)}}^{(3,4)}\right)^{\top} \tilde{\boldsymbol{C}}_{i t}^{(i)}\right)$ and $\boldsymbol{N}_{i t}^{(i)}=\operatorname{Mat}^{\left(F^{(i)} \times F^{(i)}, F^{(i)}\right)}\left(\boldsymbol{M}_{i t}^{(i)}\right)$. Note that, $\operatorname{Mat}^{I \times J, K}$ is the block matrix rearrangement operator of a $(I K \times$ $J)$ matrix, such as $\mathrm{Mat}^{(I \times J, K)}\left(\left[\boldsymbol{Z}_{1}^{\top}, \boldsymbol{Z}_{2}^{\top}, \cdots, \boldsymbol{Z}_{K}^{\top}\right]^{\top}\right)=$ $\left[\boldsymbol{Z}_{1}, \boldsymbol{Z}_{2}, \cdots, \boldsymbol{Z}_{K}\right]$, with $K$ blocks of size $(I \times P)$. A matrix computation of the previous coefficients is performed allowing for an effective implementation in matrix programming environments. The first solution is $\tilde{B}_{m n}^{(i)}=0$ and for the others, we can search by computing the positive roots of a third degree polynomial defined by:

$$
\gamma_{\tilde{A}_{m n}^{(i)}}\left(\tilde{A}_{m n}^{(i)}\right)=\left(\tilde{A}_{m n}^{(i)}\right)^{3}+\frac{\alpha_{3}}{\alpha_{7}} \tilde{A}_{m n}^{(i)}+\frac{\alpha_{1}}{\alpha_{7}}
$$

with $\tilde{A}_{m n}^{(i)}=\left(\tilde{B}_{m n}^{(i)}\right)^{2}$. Then, an analytical root can be computed using Cardono's method and choosing the positive root, which minimizes the cost function, $f_{\tilde{B}_{m n}^{(i)}}^{\prime}$. This step is achieved for all components of $\boldsymbol{B}_{i t+1}^{(i)}$. Then, the solution of the second subproblem is well-known and given by $\tilde{\boldsymbol{C}}_{i t+1}^{(i)}=\boldsymbol{T}_{\tilde{\boldsymbol{x}}^{(i)}}^{(3,4)}\left(\left(\tilde{\boldsymbol{A}}_{i t+1}^{(i)} \odot \tilde{\boldsymbol{A}}_{i t+1}^{(i)}\right)^{\sharp}\right)^{\top}[40]$.

b) Line search procedure: The ALS procedure has some known drawbacks, as slow convergence, in the context of ill-conditioned factors or high collinearity, and as sensitivity to initialization. The algorithm can stay trapped in a local minimum or cycle [41], [42]. Line search scheme has been used in order to exit from them and so, to accelerate the ALS algorithm [42]-[46]. It consists of the linear interpolation of the loading matrices, $\tilde{\boldsymbol{B}}^{(i)}$ and $\tilde{\boldsymbol{C}}^{(i)}$, from their previous estimates:

$$
\begin{aligned}
& \tilde{\boldsymbol{B}}_{n e w}^{(i)}=\tilde{\boldsymbol{B}}_{i t-1}^{(i)}+\mu_{i t}^{\tilde{B}^{(i)}} \boldsymbol{G}_{i t}^{\tilde{B}^{(i)}} \\
& \tilde{\boldsymbol{C}}_{n e w}^{(i)}=\tilde{\boldsymbol{C}}_{i t-1}^{(i)}+\mu_{i t}^{\tilde{C}^{(i)}} \boldsymbol{G}_{i t}^{\tilde{C}^{(i)}}
\end{aligned}
$$

where $\boldsymbol{G}_{i t}^{\tilde{B}^{(i)}}=\tilde{\boldsymbol{B}}_{i t+1}^{(i)}-\tilde{\boldsymbol{B}}_{i t}^{(i)}$ and $\boldsymbol{G}_{i t}^{\tilde{C}^{(i)}}=\tilde{\boldsymbol{C}}_{i t+1}^{(i)}-\tilde{\boldsymbol{C}}_{i t}^{(i)}$ represent the search directions or the directions of the cycle, computed by the ALS procedure, and where $\mu_{i t}^{\tilde{B}^{(i)}}$ and $\mu_{i t}^{\tilde{C}^{(i)}}$ are the stepsizes along those directions. The line search step is performed before applying the ALS iteration and the interpolation matrices, $\tilde{\boldsymbol{B}}_{\text {new }}^{(i)}$ and $\tilde{\boldsymbol{C}}_{\text {new }}^{(i)}$, are used as starting values for the
ALS update rules, at the iteration $i t$, instead of $\tilde{\boldsymbol{B}}_{i t}^{(i)}$ and $\tilde{\boldsymbol{C}}_{i t}^{(i)}$. After inserting the update rules of $\tilde{\boldsymbol{B}}^{(i)}$ and $\tilde{\boldsymbol{C}}^{(i)}$ in (10), both stepsizes $\mu_{i t}^{\tilde{B}^{(i)}}$ and $\mu_{i t}^{\tilde{C}^{(i)}}$ can be computed by minimizing the following function:

$$
\varphi\left(\mu^{\tilde{B}^{(i)}}, \mu^{\tilde{C}^{(i)}}\right)=\left\|\boldsymbol{T}_{\tilde{\boldsymbol{x}}^{(i)}}^{(3,4)}-\tilde{\boldsymbol{C}}_{n e w}^{(i)}\left(\left(\tilde{\boldsymbol{A}}_{\text {new }}^{(i)}\right)^{\odot 2}\right)^{\mathrm{T}}\right\|_{F}^{2}
$$

w.r.t. $\mu^{\tilde{B}^{(i)}}$ and $\mu^{\tilde{C}^{(i)}}$ and where $\tilde{\boldsymbol{A}}_{\text {new }}^{(i)}=\left(\tilde{\boldsymbol{B}}_{\text {new }}^{(i)}\right)^{\diamond 2}$. This can be done approximately or exactly [41], [43]. We propose an optimal procedure, allowing to accelerate maximally the convergence. It searches the optimal stepsizes that corresponds to the real roots of a polynomial defined in equation (14). The details of such a procedure are given in in Appendix B. Nevertheless, this procedure can considerably increase the ALS numerical complexity per iteration. Then, to have a good compromise between effectiveness and numerical complexity, it is better to calculate the optimal stepsizes, $\mu_{i t}^{\tilde{B}}$ and $\mu_{i t}^{\tilde{C}}$, every $k$ iterations with $k>1$. Finally, after the ELSALS ${ }^{\text {square }}$ step, the vector basis $\boldsymbol{A}^{(i)}$ is identified as follows: $\boldsymbol{A}^{(i)}=\left(\boldsymbol{W}^{(i)}\right)^{-\mathrm{T}} \tilde{\boldsymbol{A}}^{(i)}$, with $\tilde{\boldsymbol{A}}^{(i)}=\left(\tilde{\boldsymbol{B}}^{(i)}\right)^{\diamond 2}$ and $\boldsymbol{W}^{(i)}$ computed in the section III-B1.

\section{DATABASE AND VALIDATION RESULTS}

\section{A. Database}

A total of 87 patients treated for prostate cancer with IMRT is used to evaluate our classification procedure. The used treatment planning system is Pinnacle V7.4 (Philips Medical System, Madison, WI). The total prescribed dose is 46 Gy to the seminal vesicles delivered in 4.6 weeks, and 80 Gy to the prostate delivered in 8 weeks. The standard fractionation was of $2 \mathrm{~Gy}$ per fraction. The whole treatment and dose constraints are complied with GETUG 06 recommendations as described in [47]. The constraints are a maximal dose and a V72 $\mathrm{Gy}^{1}$ lower than $76 \mathrm{~Gy}$ and $25 \%$ for the rectal wall. The size of the images is $512 \times 512$ pixels in the axial plane, with $1 \mathrm{~mm}$ image resolution and $2 \mathrm{~mm}$ slice thickness. For each patient, the organs are manually contours by the same expert, following the same standard clinical protocol in radiotherapy. The expert contoured the clinical target (prostate and seminal vesicles) and the organs at risks ( the bladder and the rectum). For each patient, the prescribed dose is computed in a standard treatment planning system step and then resampled into the CT native space. The prostate received homogeneously 80 Gy while the nearby organs at risks received lesser and heterogeneous dose. The delivery was guidedF by means of an IGRT protocol, with cone beam CT images or two orthogonal images ( $\mathrm{kV}$ or MV imaging devices), using gold fiducial markers in $57 \%$ of patients. The events were defined as rectal bleeding ( $\geq$ Grade 1), at least one episode occurring between 6 months and 2 years after Radiation Therapy. Patients with a history of hemorrhoids were not allowed to be scored as Grade 1 bleeding. Rectal toxicity was scored according to the Common Terminology Criteria for Adverse Events (CTCAE) version 3.0. Out of 87 patients, 13 presented rectal bleeding ( $\geq$ grade1) with a follow-up time of two years.

\footnotetext{
${ }^{1}$ (volume receiving at least $72 \mathrm{~Gy}$ )
} 


\section{B. Validation}

1) Evaluation scheme: Because of the reduced number of patients in our database (especially those suffering from rectal bleeding, 13 patients), a leave-one-out cross validation is performed to evaluate the proposed classification method. A 3D individual pDD is extracted (validation data) and our method described in section II is applied to the 86 remaining 3DpDD (training data). This is repeated such that each patient is used as a test sample. The performance of the method is evaluated in terms of Sensitivity $(S e)$ and Specificity $(S p)$. The $S e$ value assesses the probability of patients with rectal bleeding being correctly classified and the $S p$ value corresponds to the probability of other patients who are correctly identified as without rectal bleeding.

2) Results: In order to study the performance of the proposed procedure, we first discuss the impact of the ranks $F^{(1)}$ and $F^{(2)}$ of $\boldsymbol{A}^{(1)}$ and $\boldsymbol{A}^{(2)}$. Then we evaluate the interest of selecting the more informative features, i.e. $\boldsymbol{E}_{k_{\mathrm{opt}}}^{(1)}$ and $\boldsymbol{E}_{\ell_{\mathrm{opt}}}^{(2)}$. Eventually, a comparative study with some classical methods is proposed.

a) Influence of the ranks $F^{(1)}$ and $F^{(2)}$ : Figures 2(a) and 2(b) show the $S e$ and $S p$ values as a function of $F^{(1)}$ and $F^{(2)}$, varying from 3 to 7 . In this experiment, the whole vector bases derived from the SN-ICA algorithm are used. In other word we assume that $\boldsymbol{E}_{\mathrm{opt}}^{(i)}=\boldsymbol{A}^{(i)}, i=\{1,2\}$. We remark that, for the high values of $F^{(1)}\left(5 \leq F^{(1)} \leq 7\right)$ and $F^{(2)}\left(3 \leq F^{(2)} \leq 4\right)$, all patients are classified in the group of rectal bleeding: $S e=1$ and $S p \simeq 0$. In contrary, when $F^{(1)}$ is low $\left(3 \leq F^{(1)} \leq 4\right)$, we obtain $S e \simeq 0$ and $S p=1$ (all patients are viewed as no rectal bleeding ones). We also observe that, for $6 \leq F^{(1)} \leq 7$ and $4 \leq F^{(2)} \leq 5$, we obtained the quite good results. The best trade off between $S e$ and $S p$ values seems to be achieved for $\left(F^{(1)}, F^{(2)}\right)=(7,5)$ : only 2 patients with rectal bleeding $(S e=0.84)$ and 2 patients without rectal bleeding $(S p=0.97)$ are not well classified.

b) Influence of feature selection: In this experiment, the procedure of the feature selection is evaluated. Figures 3(a) and 3(b) display the $S e$ and $S p$ values when varying $F^{(1)}$ and $F^{(2)}$ and for $\left(R^{(1)}, R^{(2)}\right)=(1,1)$ (i.e. only one vector of two bases $\boldsymbol{A}^{(1)}$ and $\boldsymbol{A}^{(2)}$ are selected to form the optimal vector subbases $\boldsymbol{E}_{k_{\mathrm{opt}}}^{(1)}$ and $\boldsymbol{E}_{\ell_{\mathrm{opt}}}^{(2)}$ ). We show that $S p$ is equal to 1 whatever the number of ranks, $F_{(1)}$ and $F_{(2)}$. In other words, all patients without rectal bleeding are well classified. The procedure allows to perfectly classify the two groups $(S e=1$ and $S p=1$ ) for many couples of ranks. More particularly, it is interesting to note that for $\left(F_{(1)}, F_{(2)}\right)=(4,4)$, estimated by the proposed nonnegative compression algorithm [36] (section III-B1), the obtained performance is also perfect.

c) Comparison with classical algorithms: The proposed SN-ICA algorithm was also compared to: i) four unsupervised classification approaches, namely the PCA-based method presented in [26], a classical ICA-based algorithm [48], a Nonnegative Matrix Factorization (NMF) based approach [49] and a K-means clustering algorithm [50], and ii) two supervised methods, namely Linear Discriminant Analysis (LDA) [51] and Support Vector Machine (SVM) [52]. All these methods also take the advantage of the spatial information through the use of the registered 3DpDD. The obtained results (table I) show that the SN-ICA classification method outperforms all the other methods.

TABLE I

COMPARISON OF UNSUPERVISED CLASSIFICATION TECHNIQUES AND SUPERVISED APPROACHES

\begin{tabular}{|c||c||c|}
\hline Methods & Sensitivity $(\mathrm{Se})$ & Specificity $(\mathrm{Sp})$ \\
\hline \hline SN-ICA & 1 & 1 \\
\hline Classical ICA & 0.28 & 1 \\
\hline NMF & 1 & 0.42 \\
\hline PCA & 0.76 & 1 \\
\hline LDA & 0.53 & 0.64 \\
\hline SVM & 0.23 & 0.81 \\
\hline K-means & 0.46 & 0.35 \\
\hline
\end{tabular}

\section{DISCUSSIONS AND FUTURE WORK}

Results demonstrated the robustness of the SN-ICA algorithm for the classification of 3DpDD. Although the method was herein applied to the analysis of doses for predicting radiotherapy side-effects, it can also be directly applied to different classification problems of non-rigidly aligned 3D voxel data.

The identification of bases combined with orthogonal projection improves the results with respect to the use of only the orthogonal projections onto the raw population data $\boldsymbol{X}^{(1)}$ and $\boldsymbol{X}^{(2)}$. Indeed, using our simple classification scheme without any vector basis identification leads to very poor performances, since most of the patients were classified as rectal bleeding ( $S e=1$ and $S p=0.02$ ). In addition, all the supervised methods (which do not explicitly identify vector bases) give poor results (see table I).

Two other major contributions in this paper are: i) the consideration of non-negativity constraints together with independency, and ii) the nonnegative compression. The nonnegativity is in line with the positive character of dose, which is meaningful for this application. The SN-ICA algorithm allows for the combination of nonnegative constraints with independent assumption as opposed to PCA, classical ICA and NMF methods, which use orthogonality, independence and nonnegativity constraints, respectively. Regarding the nonnegative compression, it allows for the estimation of the best basis ranks $F^{(i)}$. Moreover, reducing the dimension of the observation space, yields an increased computational efficiency, without degrading the performance of the procedure.

Our classification criterion is based on the euclidean norm and does not require any evolutive classification method. In addition to that, a careful selection of more informative features from the computed bases is also crucial for the classification. With these data, significantly improved performances are obtained $(S e=1$ and $S p=1)$.

The proposed framework also depends on a non-rigid marching organ and CTs scans. The exploitation of euclidean distance maps from organ boundaries allowed for careful matching of the dose near to the rectal wall. Nevertheless, further work will be investigated to enhance the alignment of all structures. Another key point to consider, in future studies, 


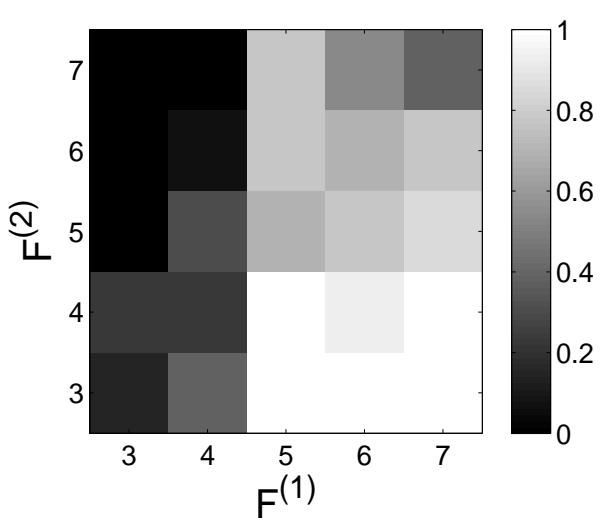

(a) $S e$ for $\left(R^{(1)}, R^{(2)}\right)$ equal to $\left(F^{(1)}, F^{(2)}\right)$

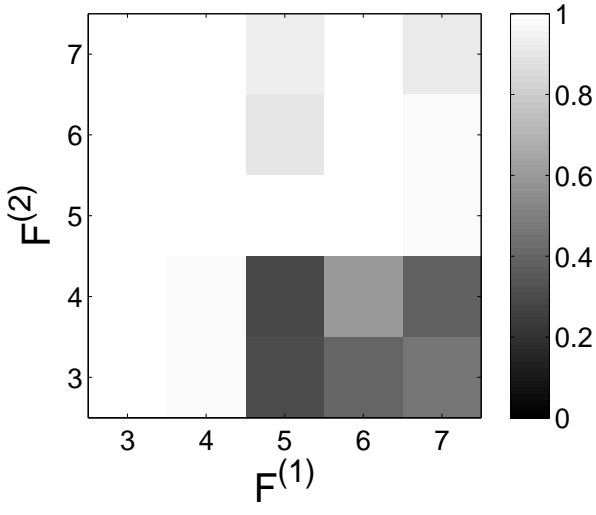

(b) $S p$ for $\left(R^{(1)}, R^{(2)}\right)$ equal to $\left(F^{(1)}, F^{(2)}\right)$

Fig. 2. Performance of the multivoxel-based Semi-Nonnegative ICA (SN-ICA) feature extraction for different couples of ranks without selection of features.

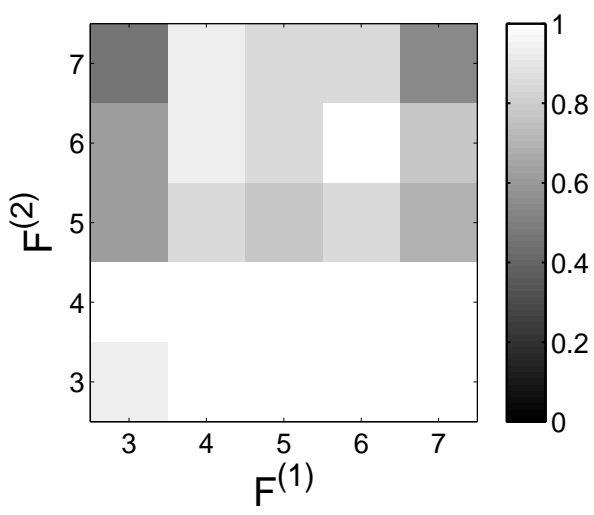

(a) Se for $\left(R^{(1)}, R^{(2)}\right)=(1,1)$

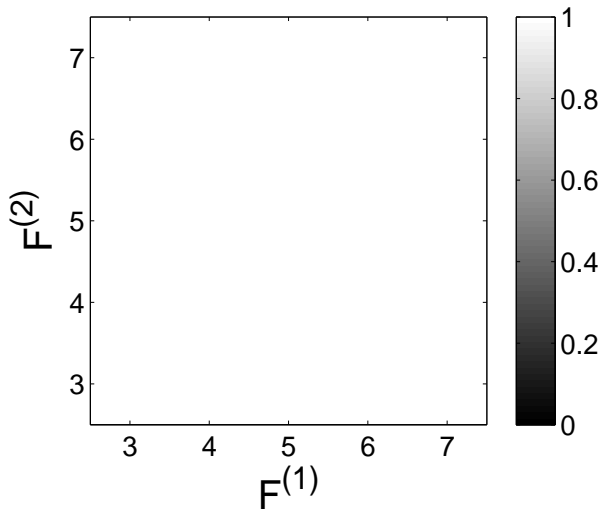

(b) $S p$ for $\left(R^{(1)}, R^{(2)}\right)=(1,1)$

Fig. 3. Performance of the multivoxel-based Semi-Nonnegative ICA (SN-ICA) feature extraction for different couples of ranks and selection of one feature.

relies on the optimal template selected from the database to be representative of a given population.

Although the results are promising in terms of performance, concerning the clinical application, the results shall be confirmed with larger databases. Toxicity prediction has been addressed by a large number of studies using DVHs [6]-[9], which differ from our proposed approach. Despite the lack of spatial accuracy, these well-established methods provide good prediction results. In future work, a thorough validation against those models will be performed. One of the issues for this comparison to be carried out lies in the need of large cohorts to estimate the population specific parameters. As opposed to them, the proposed method exploits the rich spatial information encoded in the 3DpDD yielding a perfect classification. The good performance reveals that classifying rectal bleeding and non rectal bleeding patients from a population treated for prostate cancer is possible. Clearly, in this study we don't claim that the 3DpDD is the only factor responsible of side effects. Obviously, it is well known that the toxicity is also related to specific factors of an individual [16], [17], [53],
[54], such as radio-sensitivity, personal medical history, age, concomitant treatment, etc. Further works should also consider the inclusion of these individual's clinical variables that may be involved in toxicity as an additional criterion within the classification.

\section{CONCLUSION}

In this work, we have proposed a new method for classifying patient at risk of suffering from rectal bleeding after prostate cancer radiotherapy. The proposed approach is based on a new SN-ICA algorithm, which allows for the extraction of more informative features from the 3D planning Dose Distributions (3DpDD). The method exploits the rich spatial information conveyed by the 3DpDD thanks to the determination of two bases for both rectal bleeding and non rectal bleeding patients. The classification is then achieved by orthogonally projecting a 3D individual test onto both subspaces spanned by the more informative vectors. The test patient is thus classified using an euclidean distance. A comparative study demonstrates the performance of our method, which provides a new means of 
predicting toxicity. This framework may in turn be used for improving the therapy by introducing new constraints within the IMRT planning, and the SN-ICA may also constitutes a promising procedure for processing other image applications where the nonnegative constraints and the independence assumption are verified.

\section{ACKNOWLEDGEMENT}

This work has been funded by the Institut de Recherche en Santé Publique-IreSP (France), under the Plan Cancer 20092013 Call (No. C13005N5).

\section{APPENDIX A}

\section{THE NONNEGATIVE COMPRESSION}

The shearing transformation for a pair of columns of $\boldsymbol{W}^{(i)}$, called $\boldsymbol{w}_{k}^{(i)}$ and $\boldsymbol{w}_{\ell}^{(i)}$, is defined as follows:

$$
\left[\widehat{\boldsymbol{w}}_{k}^{(i)}, \widehat{\boldsymbol{w}}_{\ell}^{(i)}\right]=\left[\boldsymbol{w}_{k}^{(i)}, \boldsymbol{w}_{\ell}^{(i)}\right]\left[\begin{array}{cc}
1 & 0 \\
\lambda & 1
\end{array}\right]
$$

where the parameter $\lambda$ is called a shear factor. Noting that $\widehat{\boldsymbol{w}}_{\ell}^{(i)}$ remains unchanged, we consider the following negativeness measure criterion [38]:

$$
J(\lambda)=\frac{1}{2} \sum_{j}^{I}\left(\widehat{W}_{j, k}^{(i)}\right)^{2} \mathbb{1}_{\widehat{W}_{j, k}^{(i)}<0}
$$

where $\mathbb{1}_{\alpha<0}=\left\{\begin{array}{ll}1 & \text { if } \alpha<0 \\ 0 & \text { otherwise }\end{array}\right.$ and $\widehat{W}_{j, k}^{(i)}$ is the $(j, k)$ th component of $\widehat{\boldsymbol{W}}^{(i)}$. The purpose is to find a $\lambda$ which minimizes the total sum of squares of negative elements in $\widehat{\boldsymbol{w}}_{k}^{(i)}(16)$. The global optimum $\lambda^{\text {opt }}$ is the root of the derivative of $J(\lambda)$. However, such a $\lambda^{o p t}$ is difficult to obtain analytically, since the heaviside-step-like function $\mathbb{1}_{\alpha<0}$ exists in (16). We propose to compute $\lambda$ iteratively by a Newton's method. At iteration $i t$, the update rule of $\lambda$ is given by:

$$
\lambda_{i t}=\lambda_{i t-1}-\left(\frac{\partial^{2} J\left(\lambda_{i t-1}\right)}{\partial \lambda_{i t-1}^{2}}\right)^{-1} \frac{\partial J\left(\lambda_{i t-1}\right)}{\partial \lambda_{i t-1}}
$$

where $\lambda_{i t-1}$ is the solution at the previous iteration, $\partial J\left(\lambda_{i t-1}\right) / \partial \lambda_{i t-1}$ and $\partial^{2} J\left(\lambda_{i t-1}\right) / \partial \lambda_{i t-1}^{2}$ are the first and second order derivatives of $J$ w.r.t $\lambda_{i t-1}$ which are given as follows, respectively:

$$
\frac{\partial J\left(\lambda_{i t-1}\right)}{\partial \lambda_{i t-1}}=\sum_{j}^{I}\left(W_{j, k}^{(i)}+\lambda_{i t-1} W_{j, \ell}^{(i)}\right) W_{j, \ell}^{(i)} \mathbb{1}_{\left(W_{j, k}^{(i)}+\lambda_{i t-1} W_{j, \ell}^{(i)}\right)<0}
$$

and

$$
\frac{\partial^{2} J\left(\lambda_{i t-1}\right)}{\partial \lambda_{i t-1}^{2}}=\sum_{j}^{I}\left(W_{j, \ell}^{(i)}\right)^{2} \mathbb{1}_{\left(W_{j, k}^{(i)}+\lambda_{i t-1} W_{j, \ell}^{(i)}\right)<0}
$$

$\partial^{2} J\left(\lambda_{i t-1}\right) / \partial \lambda_{i t-1}^{2}=0$ indicates that all the elements of $\widehat{\boldsymbol{w}}_{k}^{(i)}$ have the same sign. Then the above procedure is repeated with the next columns pair. Several sweeps of this sequential optimization procedure are necessary to ensure convergence. More details can be found in [55].

\section{APPENDIX B}

\section{THE COMPUTATION OF THE MATRIX $\boldsymbol{F}$}

The function $\varphi$ (14) can be rewritten as follows:

$$
\begin{aligned}
& \varphi\left(\mu^{\tilde{B}^{(i)}}, \mu^{\tilde{C}^{(i)}}\right)=\| \boldsymbol{F}_{0}+\boldsymbol{F}_{1} \mu^{\tilde{B}^{(i)}}+\boldsymbol{F}_{2}\left(\mu^{\tilde{B}^{(i)}}\right)^{2}+ \\
& \boldsymbol{F}_{3}\left(\mu^{\tilde{B}^{(i)}}\right)^{3}+\boldsymbol{F}_{4}\left(\mu^{\tilde{B}^{(i)}}\right)^{4}+\boldsymbol{F}_{5} \mu^{\tilde{C}^{(i)}}+\boldsymbol{F}_{6} \mu^{\tilde{C}^{(i)}}\left(\mu^{\tilde{B}^{(i)}}\right)+ \\
& \boldsymbol{F}_{7} \mu^{\tilde{C}^{(i)}}\left(\mu^{\tilde{B}^{(i)}}\right)^{2}+\boldsymbol{F}_{8} \mu^{\tilde{C}^{(i)}}\left(\mu^{\tilde{B}^{(i)}}\right)^{3}+\boldsymbol{F}_{9} \mu^{\tilde{C}^{(i)}}\left(\mu^{\tilde{B}^{(i)}}\right)^{4} \|_{F}^{2}
\end{aligned}
$$

where:

$$
\begin{aligned}
& \boldsymbol{F}_{0}=\boldsymbol{T}_{\tilde{\boldsymbol{x}}^{(i)}}^{(3,4)}-\tilde{\boldsymbol{C}}_{i t-1}^{(i)} \boldsymbol{E}_{0} \quad \boldsymbol{F}_{1}=-\tilde{\boldsymbol{C}}_{i t-1}^{(i)} \boldsymbol{E}_{1} \\
& \boldsymbol{F}_{2}=-\tilde{\boldsymbol{C}}_{i t-1}^{(i)} \boldsymbol{E}_{2} \quad \boldsymbol{F}_{3}=-\tilde{\boldsymbol{C}}_{i t-1}^{(i)} \boldsymbol{E}_{3} \\
& \boldsymbol{F}_{4}=-\tilde{\boldsymbol{C}}_{i t-1}^{(i)} \boldsymbol{E}_{4} \quad \boldsymbol{F}_{5}=-\boldsymbol{G}_{i t}^{\tilde{C}^{(i)}} \boldsymbol{E}_{0} \\
& \boldsymbol{F}_{6}=-\boldsymbol{G}_{i t}^{\tilde{C}^{(i)}} \boldsymbol{E}_{1} \quad \boldsymbol{F}_{7}=-\boldsymbol{G}_{i t}^{\tilde{C}^{(i)}} \boldsymbol{E}_{2} \\
& \boldsymbol{F}_{8}=-\boldsymbol{G}_{i t}^{\tilde{C}^{(i)}} \boldsymbol{E}_{3} \quad \boldsymbol{F}_{9}=-\boldsymbol{G}_{i t}^{\tilde{C}^{(i)}} \boldsymbol{E}_{4}
\end{aligned}
$$

with:

$$
\begin{aligned}
& \boldsymbol{E}_{0}=\left(\boldsymbol{K}_{0} \odot \boldsymbol{K}_{0}\right)^{\mathrm{T}} \quad \boldsymbol{E}_{1}=\left(\boldsymbol{K}_{0} \odot \boldsymbol{K}_{1}+\boldsymbol{K}_{1} \odot \boldsymbol{K}_{0}\right)^{\mathrm{T}} \\
& \boldsymbol{E}_{2}=\left(\boldsymbol{K}_{0} \odot \boldsymbol{K}_{2}+\boldsymbol{K}_{1} \odot \boldsymbol{K}_{1}+\boldsymbol{K}_{2} \odot \boldsymbol{K}_{0}\right)^{\top} \\
& \boldsymbol{E}_{3}=\left(\boldsymbol{K}_{1} \odot \boldsymbol{K}_{2}+\boldsymbol{K}_{2} \odot \boldsymbol{K}_{1}\right)^{\top} \quad \boldsymbol{E}_{4}=\left(\boldsymbol{K}_{2} \odot \boldsymbol{K}_{2}\right)^{\top}
\end{aligned}
$$

and finally:

$$
\begin{gathered}
\boldsymbol{K}_{0}=\tilde{\boldsymbol{A}}_{i t-1}^{(i)}=\left(\tilde{\boldsymbol{B}}_{i t-1}^{(i)}\right)^{\diamond 2} \quad \boldsymbol{K}_{2}=\left(\boldsymbol{G}_{i t}^{\tilde{B}^{(i)}}\right)^{\diamond 2} \\
\boldsymbol{K}_{1}=\tilde{\boldsymbol{B}}_{i t-1}^{(i)} \diamond \boldsymbol{G}_{\tilde{B}^{(i)}}+\boldsymbol{G}_{\tilde{B}^{(i)}} \diamond \tilde{\boldsymbol{B}}_{i t-1}^{(i)}
\end{gathered}
$$

In order to calculate $\mu_{i t}^{\tilde{B}^{(i)}}$ and $\mu_{i t}^{\tilde{C}^{(i)}}$, the function $\varphi$ can be reduced to a compact form as follows:

$$
\varphi\left(\mu^{\tilde{B}^{(i)}}, \mu^{\tilde{C}^{(i)}}\right)=\|\boldsymbol{F} \boldsymbol{u}\|_{F}^{2}=\boldsymbol{u}^{\top} \boldsymbol{F}^{\top} \boldsymbol{F} \boldsymbol{u}
$$

where $\boldsymbol{F}=\left[\operatorname{vec}\left[\boldsymbol{F}_{9}\right], \operatorname{vec}\left[\boldsymbol{F}_{8}\right], \ldots, \operatorname{vec}\left[\boldsymbol{F}_{1}\right], \operatorname{vec}\left[\boldsymbol{F}_{0}\right]\right]$ is a $\left(\left(F^{(i)}\right)^{4}+\left(F^{(i)}\right)^{3} \times 10\right)$ matrix, and $\boldsymbol{u}=\left[\mu^{\tilde{C}^{(i)}}\left(\mu^{\tilde{B}^{(i)}}\right)^{4}\right.$, $\mu^{\tilde{C}^{(i)}}\left(\mu^{\tilde{B}^{(i)}}\right)^{3}, \mu^{\tilde{C}^{(i)}}\left(\mu^{\tilde{B}^{(i)}}\right)^{2}, \mu^{\tilde{C}^{(i)}}\left(\mu^{\tilde{B}^{(i)}}\right), \mu^{\tilde{C}^{(i)}},\left(\mu^{\tilde{B}^{(i)}}\right)^{4}$, $\left.\left(\mu^{\tilde{B}^{(i)}}\right)^{3},\left(\mu^{\tilde{B}^{(i)}}\right)^{2},\left(\mu^{\tilde{B}^{(i)}}\right), 1\right]^{\top}$ is a 10 -dimensional vector.

The objective function $\varphi$ is a second degree polynomial in $\mu^{\tilde{C}^{(i)}}$. The optimal stepsize $\mu_{i t}^{\tilde{C}^{(i)}}$ is achieved by vanishing the partial derivative w.r.t. $\mu^{\tilde{C}^{(i)}}$, then $\mu_{i t}^{\tilde{C}^{(i)}}$ is equal to a rational function in $\mu^{\tilde{B}^{(i)}}$. The expression of $\mu_{i t}^{\tilde{C}^{(i)}}$ is injected in the equation $\partial \varphi / \partial \mu^{\tilde{B}^{(i)}}=0$. Consequently, the global minimum $\mu_{i t}^{\tilde{C}^{(i)}}$ can be computed by rooting the numerator of $\partial \varphi / \partial \mu^{\tilde{B}^{(i)}}$, which is a 24-th degree polynomial in $\mu^{\tilde{B}^{(i)}}$ and selecting the root yielding the smallest value of the objective function $\varphi$. Then, we infer $\mu_{i t}^{\tilde{C}^{(i)}}$ from $\mu_{i t}^{\tilde{B}^{(i)}}$.

\section{REFERENCES}

[1] A. L. ZIEMAN, K. BAE, J. D. SLATER, W. U. SHIPLEY, J. A. EFSTATHIOU, J. J. COEN, D. A. BUSH, M. LUNT, D. Y. SPIEGEL, R. SKOWRONSKI, B. R. JABOLA, and C. J. ROSSI, "Randomized trial comparing conventional-dose with high-dose conformal radiation therapy in early-stage adenocarcinoma of the prostate: long-term results from proton radiation oncoloGy group/american college of radioloGy 95-09.” J Clin Oncol, vol. 28, no. 7, pp. 1106-1111, Mar 2010.

[2] R. D. CREVOISIER, P. POMMIER, J. BACHAUD, G. CREHANGE, C. BOUTRY, B. CHAUVET, T. NGUYEN, A. LAGRANGE, M. AUBELLE, and J. LAGRANGE, "Image-guided radiation therapy (IGRT) in prostate cancer: Preliminary results in prostate registration and acute toxicity of a randomized study," International Journal of Radiation OncoloGy BioloGy Physics, vol. 75, no. 3, Supplement, pp. S99-, 2009. 
[3] V. FONTEYNE, G. VILLEIRS, B. SPELEERS, W. D. NEVE, C. D. WATGER, N. LUMEN, and G. D. MEERLEER, "Intensity-modulated radiotherapy as primary therapy for prostate cancer: report on acute toxicity after dose escalation with simultaneous integrated boost to intraprostatic lesion." Int J Radiat Oncol Biol Phys, vol. 72, no. 3, pp. 799-807, Nov 2008.

[4] C. FIORINO, T. RANCATI, and R. VALDALGNI, "Predictive models of toxicity in external radiotherapy: dosimetric issues." Cancer, vol. 115, no. 13 Suppl, pp. 3135-3140, Jul 2009.

[5] J. Y. TING, X. WU, J. A. FIEDLER, C. YANG, M. L. WATZICH, and A. MARKOE, "Dose-volume histograms for bladder and rectum." Int J Radiat Oncol Biol Phys, vol. 38, no. 5, pp. 1105-1111, Jul 1997.

[6] S. WACHTER, N. GERSTNER, G. GOLDNER, R. PÖTZI, A. WAMBERSIE, and R. PÖTTER, "Rectal sequelae after conformal radiotherapy of prostate cancer: dose-volume histograms as predictive factors." Radiother Oncol, vol. 59, no. 1, pp. 65-70, Apr 2001.

[7] G. N. GRIGOROV, J. C. L. CHOW, L. GRIGOROV, R. JIANG, and R. B. BARNETT, "IMRT: improvement in treatment planning efficiency using ntcp calculation independent of the dose-volume-histogram." Med Phys, vol. 33, no. 5, pp. 1250-1258, May 2006.

[8] R. CAMBRIA, B. A. JERECZEK-FOSSA, F. CATTANI, C. GARIBALDI, D. ZERINI, C. FODOR, F. SERAFINI, G. PEDROLI, and R. ORECCHIA, "Evaluation of late rectal toxicity after conformal radiotherapy for prostate cancer: a comparison between dose-volume constraints and ntcp use." Strahlenther Onkol, vol. 185, no. 6, pp. 384-389, Jun 2009.

[9] I. JENSEN, J. CARL, B. LUND, E. H. LARSEN, and J. NIELSEN, "Radiobiological impact of reduced margins and treatment technique for prostate cancer in terms of tumor control probability (TCP) and normal tissue complication probability (NTCP).” Med Dosim, May 2010.

[10] C. FIORINO, C. COZZARINI, V. VAVASSORI, G. SANGUINETI, C. BIANCHI, G. M. CATTANEO, F. FOPPIANO, A. MAGLI, and A. PIAZZOLLA, "Relationships between DVHs and late rectal bleeding after radiotherapy for prostate cancer: analysis of a large group of patients pooled from three institutions." Radiother Oncol, vol. 64, no. 1, pp. 1-12, Jul 2002.

[11] M. SCHWARZ, J. V. LEBESQUE, B. J. MIJNHEER, and E. M. F. DAMEN, "Sensitivity of treatment plan optimisation for prostate cancer using the equivalent uniform dose (eud) with respect to the rectal wall volume parameter." Radiother Oncol, vol. 73, no. 2, pp. 209-218, Nov 2004.

[12] T. RANCATI, C. FIORINO, G. GAGLIARDI, G. M. CATTANEO, G. SANGUINETI, V. C. BORCA, C. COZZARINI, G. FELLIN, F. FOPPIANO, G. GIRELLI, L. MENEGOTTI, A. PIAZZOLLA, V. VAVASSORI, and R. VALDAGNI, "Fitting late rectal bleeding data using different NTCP models: results from an italian multi-centric study (airopros0101)." Radiother Oncol, vol. 73, no. 1, pp. 21-32, Oct 2004.

[13] S. T. H. PEETERS, M. S. HOOGEMAN, W. D. HEEMSBERGEN, A. A. M. HART, P. C. M. KOPER, and J. V. LEBESQUE, "Rectal bleeding, fecal incontinence, and high stool frequency after conformal radiotherapy for prostate cancer: normal tissue complication probability modeling." Int J Radiat Oncol Biol Phys, vol. 66, no. 1, pp. 11-19, Sep 2006.

[14] S. MARZI, G. ARCANGELI, B. SARACINO, M. G. PETRONGARI, V. BRUZZANITI, G. IACCARINO, V. LANDONI, A. SORIANI, and M. BENASSI, "Relationships between rectal wall dose-volume constraints and radiobiologic indices of toxicity for patients with prostate cancer." Int J Radiat Oncol Biol Phys, vol. 68, no. 1, pp. 41-49, May 2007.

[15] C. FIORINO, R. VALDAGNI, T. RANCATI, and G. SANGUINETI, "Dose-volume effects for normal tissues in external radiotherapy: pelvis." Radiother Oncol, vol. 93, no. 2, pp. 153-167, Nov 2009.

[16] G. DEFRAENE, L. V. D. BERGH, A. AL-MAMGANI, K. HAUSTERMANS, W. HEEMSBERGEN, F. V. D. HEUVEL, and J. LEBESQUE, "The benefits of including clinical factors in rectal normal tissue complication probability modeling after radiotherapy for prostate cancer." Int J Radiat Oncol Biol Phys, vol. 82, no. 3, pp. 1233-1242, 2012.

[17] L. CELlA, V. D. AVINO, R. LIUZZI, M. CONSON, F. DORIA, A. FAIELLA, F. LOFFREDO, M. SALVATORE, and R. PACELLI, "Multivariate normal tissue complication probability modeling of gastrointestinal toxicity after external beam radiotherapy for localized prostate cancer," Radiation OncoloGy, vol. 87, no. 2, pp. 304-310, 2013.

[18] F. BUETTNER, S. L. GULLIFORD, S. WEBB, M. SYDES, D. P. DEARNALEY, and M. PARTRIDGE, "Assessing correlations between the spatial distribution of the dose to the rectal wall and late rectal toxicity after prostate radiotherapy: an analysis of data from the MRC
RT01 trial (isrctn 47772397)," Physics in Medicine and BioloGy, vol. 54, no. 21 , p. $6535,2009$.

[19] F. BUETTNER, S. L. GULLIFORD, S. WEBB, and M. PARTRIDGE, "Modeling late rectal toxicities based on a parameterized representation of the 3d dose distribution," Physics in Medicine and BioloGy, vol. 56, no. 7, p. 2103, 2011. [Online]. Available: http://stacks.iop.org/0031$9155 / 56 / \mathrm{i}=7 / \mathrm{a}=013$

[20] G. DREAN, O. ACOSTA, A. SIMON, R. D. CREVOISIER, and P. HAIGRON, "Evaluation of inter-individual pelvic CT-scans registration," IRBM, vol. 32, no. 5, pp. 288-292, Nov 2011.

[21] G. DREAN, O. ACOSTA, A. SIMON, R. DE CREVOISIER, and P. HAIGRON, "Inter-individual organ-driven CT registration for dose mapping in prostate cancer radiotherapy," in Biomedical Imaging (ISBI), 2012 9th IEEE International Symposium on, may 2012, pp. 370-373.

[22] O. ACOSTA, G. DREAN, J. OSPINA, A. SIMON, P. HAIGRON, C. LAFOND, and R. D. CREVOISIER, "Voxel-based population analysis for correlating local dose and rectal toxicity in prostate cancer radiotherapy," Physics in Medicine and BioloGy, vol. 58, no. 8, 2013.

[23] G. DREAN, O. ACOSTA, A. SIMON, G. CAZOULAT, P. HAIGRON, K. GNEP, J. ZHU, O. HENRY, and R. D. CREVOISIER, "Voxel-based analysis of dose for toxicity prediction in prostate cancer radiation therapy," American Society for Radiation OncoloGy, 2012.

[24] J. FRIPP, P. BOURGEAT, O. ACOSTA, P. RAGINA, M. MODAT, K. PIKE, G. JONES, G. O'KEEPE, C. MASTERS, and D. AMES, "Appearance modeling of 11C PiB PET images: characterizing amyloid deposition in alzheimer's disease, mild cognitive impairment and healthy aging." Neuroimage, vol. 43, no. 3, p. 430, 2008.

[25] B. CHEN, O. ACOSTA, A. KACHENOURA, J. OSPINA, G. DREAN, A. SIMON, J. BELLANGER, P. HAIGRON, and R. D. CREVOISIER, "Spatial characterization and classification of rectal bleeding in prostate cancer radiotherapy with a voxel-based principal components analysis model for 3d dose distribution," in Prostate Cancer Imaging Workshop, MICCAI 2011, Toronto, CA, Sep 2011, Lecture Notes in Computer Science, vol. 6963, 2011, pp. 60-69.

[26] A. FARGEAS, A. KACHENOURA, O. ACOSTA, L. ALBERA, G. DREAN, and R. D. CREVOISIER, "Feature extraction and classification for rectal bleeding in prostate cancer radiotherapy: A pca based method," IRBM journal, 2013.

[27] H. PARASTAR, M. JALALI-HERAVI, and R. TAULER, "Is independent component analysis appropriate for multivariate resolution in analytical chemistry ?" J. Analytical Chemistry, vol. 31, pp. 134-143, 2012.

[28] L. AlBERA, A. KACHENOURA, P. COMON, A. KARFOUL, F. WENDLING, L. SENHADJI, and I. MERLET, "ICA-based eeg denoising: a comparative analysis of fifteen methods," in Special Issue of the Bulletin of the Polish Academy of Sciences, vol. 60, no. 3, pp. 407-418, 2012

[29] A. KACHENOURA, L. ALBERA, L. SENHADJI, and P. COMON, "ICA: a potential tool for BCI systems," IEEE Signal Processing Magazine, special issue on Brain-Computer Interfaces, vol. 25, no. 1, pp. 57-68, January 2008 .

[30] L. ALBERA, P. COMON, L. C. PARRA, A. KARFOUL, A. KACHENOURA, and L. SENHADJI, "ICA and biomedical applications," in Handbook of Blind Source Separation, P. C. . C.Jutten, Ed. London, UK: Academic Press, 2010, ch. 18, pp. 737-777.

[31] M. S. BARTLETT, J. MOVELLAN, and T. SEJNOWSKI, "Face recognition by Independent Component Analysis," IEEE Transcations on Neural Networks, vol. 13, no. 6, pp. 1450-1464, 2002.

[32] J. PULKKINEN, A. M. HAKKINEN, N. LUNDBOM, A. PAETAU, R. A. KAUPPINEN, and Y. HILTUNEN, "Independent component analysis to proton spectroscopic imaging data to human brain tumours," European Journal of RadioloGy, vol. 56, no. 2, pp. 160-164, 2005.

[33] S. PARFAIT, P. WALKER, G. CREHANGE, X. TIXON, and J. MEITERAN, "Classification of prostate magnetic resonance spectra using support vector machine," Biomedical Signal Processing and Control, vol. 7, pp. 499-508, 2012.

[34] J. COLOIGNER, L. ALBERA, A. KACHENOURA, and L. SENHADJI, "Canonical decomposition of semi-symmetric semi-nonnegative threeway arrays," in ASILOMAR 11, IEEE Forty-fifth Asilomar Conference on Signals, Systems and Computers, Asilomar, US, CA, November 6-9 2011.

[35] J. COLOIGNER, L. ALBERA, A. KARFOUL, A. KACHENOURA, P. COMON, and L. SENHADJI., "Semi-nonnegative independent component analysis," in in LVA/ICA 10, ninth international conference on latent variable analysis and signal separation, Saint Malo, France, September 27-30 2010, pp. 612-619. 
[36] I. T. JOLLIFFE, Principal component analysis. Springer Series in Statistics, 2nd ed., 2005.

[37] M. PLUMBLEY, "Algorithms for non-negative independent component analysis," IEEE Transactions on Neural Networks, vol. 14, pp. 534-543, 2002.

[38] W. OUEDRAOGO, A. SOULOUMIAC, and C. JUTTEN, "Non-negative independent component analysis algorithm based on $2 \mathrm{~d}$ givens rotations and a newton optimization," in 9th international conference Latent Variable Analysis and Signal Separation, St. Malo, France, September 27-30 2010 .

[39] G. TOMASI and R. BRO, "A comparison of algorithms for fitting the parafac model," Computational Statistics and Data Analysis, vol. 50, no. 7 , pp. $1700-1734,2006$.

[40] P. COMON, X. LUCIANI, and A. L. F. D. ALMEIDA, "Tensor decomposition, alternating least squares and other tales," Journal of Chemometrices, vol. 23, no. 393-405, pp. 393-405, August 2009.

[41] M. RAJIH, P. COMON, and R. A. HARSHMAN, "Enhanced line search : A novel method to accelerate PARAFAC," SIAM Journal on Matrix Analysis Appl., vol. 30, no. 3, pp. 1148-1171, Sep. 2008.

[42] Y. CHEN, D. HAN, and L. QI, "New als methods with extrapolation search direction and optimal step size for complex-valued tensor decomposition," IEEE Transactions on Signal Processing, vol. 59, pp. 5888$5898,2011$.

[43] R. BRO, "Multi-way analysis in the food industry: Models, algorithms, and applications," Ph.D. dissertation, University of Amsterdam, 1998.

[44] R. A. HARSHMAN, "Foundation of PARAFAC procedure: Models and conditions for an 'explanatory' multi-mode factor analysis," UCLA working papers in Phonetics, no. 16, pp. 1-84, 1970.

[45] P. K. HOPKE, P. PAATERO, H. JIA, R. T. ROSS, and R. A. HARSHMAN, "Three-way (parafac) factor analysis: Examination and comparison of alternative computational methods as applied to ill-conditioned data," Chemometrics and Intelligent Laboratory Systems, vol. 43, no. 1-2, pp. $25-42,1998$.

[46] H. GHENNIOUI, N. Thirion-Moreau, and E. Moreau, "An optimal step size relative gradient based joint diagonalization algorithm," in IEEE International Workshop on Computational Advances in Multi-Sensor Adaptive Processing, 2009.

[47] V. BECKENDORF, S. GUERIF, E. L. PRISE, J.-M. COSSET, A. BOUGNOUX, B. CHAUVET, N. SALEM, O. CHAPET, S. BOURDAIN, J.-M. BACHAUD, P. MAINGON, J.-M. HANNOUN-LEVI, L. MALISSARD, J.-M. SIMON, P. POMMIER, M. HAY, B. DUBRAY, J.-L. LAGRANGE, E. LUPORSI, and P. BEY, "70 gy versus 80 gy in localized prostate cancer: 5-year results of getug 06 randomized trial." Int J Radiat Oncol Biol Phys, vol. 80, no. 4, pp. 1056-1063, Jul 2011.

[48] A. BELOUCHRANI, K. ABED-MERAIM, J.-F. CARDOSO, and E. MOULINES, "A blind source separation technique using secondorder statistics," IEEE Transactions On Signal Processing, vol. 45, no. 2, pp. 434-444, February 1997.

[49] C.-J. LIN, "Projected gradient methods for nonnegative matrix factorization. neural computing," Neural Computing, vol. 19, no. 10, pp. 27562779, 2007.

[50] FRALEY and A. E. RAFTERY, "How many clusters? which clustering method? answers via model-based cluster analysis," The computer journal, vol. 41, no. 8, pp. 578-588, 1998.

[51] A. M. MARTINEZ and A. C. KAK, "PCA versus LDA," IEEE Transcations on pattern anaysis and machine intelligence, vol. 23, no. 2 , pp. 228-233, 2001.

[52] N. CRISTIANINI and J. SHAWE-TAYLOR, "An introduction to support vector machines and other kernel-based learning methods." Cambridge university press, 2000.

[53] M. LIU, V. MOISEENKO, A. AGRANOVICH, A. KARVAT, W. KWAN, Z. H. SALEH, A. A. APTE, and J. O. DEASY, "Normal tissue complication probability (ntcp) modeling of late rectal bleeding following external beam radiotherapy for prostate cancer: A test of the quantec-recommended ntcp model," Acta Oncology, vol. 49, no. 7, pp. 1040-1044, 2010.

[54] T. RANCATI, C. FIORINO, G. FELLIN, V. VAVASSORI, E. CAGNA, V. C. BORCA, G. GIRELLI, L. MENEGOTTI, A. F. MONTI, F. TORTORETO, and al., "Inclusion of clinical risk factors into NTCP modelling of late rectal toxicity after high dose radiotherapy for prostate cancer," Radiotherapy and OncoloGy, vol. 100, no. 1, pp. 124-130, 2011.

[55] L. WANG, A. KACHENOURA, L. ALBERA, A. KARFOUL, H. SHU, and L. SENHADJI, "Nonnegative compression for semi-nonnegative independent component analysis," in the Eighth IEEE Sensor Array and Multichannel Signal Processing Workshop (SAM'14), no. 4, La Corogne, Spain, June 22-25 2001, pp. 13-16. 